\title{
Genetic Diversity of Mycobacterium tuberculosis Complex Isolated from Tuberculosis Patients in Bahir Dar City and Its Surroundings, Northwest Ethiopia
}

\author{
Anwar Nuru, ${ }^{1,2}$ Gezahegne Mamo, ${ }^{3}$ Adane Worku, ${ }^{1}$ Aschalew Admasu, ${ }^{4}$ \\ Girmay Medhin, ${ }^{1}$ Rembert Pieper, ${ }^{5}$ and Gobena Ameni ${ }^{1}$ \\ ${ }^{1}$ Aklilu Lemma Institute of Pathobiology, Addis Ababa University, P.O. Box 1176, Addis Ababa, Ethiopia \\ ${ }^{2}$ Faculty of Veterinary Medicine, University of Gondar, P.O. Box 346, Gondar, Ethiopia \\ ${ }^{3}$ College of Veterinary Medicine and Agriculture, Addis Ababa University, P.O. Box 34, Debre Zeit, Ethiopia \\ ${ }^{4}$ Bahir Dar Regional Health Research Laboratory Centre, P.O. Box 641, Bahir Dar, Ethiopia \\ ${ }^{5}$ J. Craig Venter Institute, 9704 Medical Center Drive, Rockville, MD, USA
}

Correspondence should be addressed to Gobena Ameni; gobenachimdi2009@yahoo.co.uk

Received 20 June 2015; Revised 8 August 2015; Accepted 17 August 2015

Academic Editor: Isabel Portugal

Copyright (C) 2015 Anwar Nuru et al. This is an open access article distributed under the Creative Commons Attribution License, which permits unrestricted use, distribution, and reproduction in any medium, provided the original work is properly cited.

\begin{abstract}
The knowledge of the diversity of strains of Mycobacterium tuberculosis complex (MTBC) species in a specific geographical region can contribute to the control of tuberculosis (TB). This study was conducted to identify the MTBC isolates to the species and spoligotype international type (SIT) level by spoligotyping. A total of $168 \mathrm{MTBC}$ isolates were recovered from TB patients, spoligotyped, and their patterns were compared with those of the strains registered in the SITVIT2 database. Of 168 isolates spoligotyped, 89 patterns were identified. Ninety-eight isolates were clustered into 19 strain groups with clustering percentage of $58.3 \%$. Forty-four strains matched the preexisting SITs in the SITVIT2 database. The dominant strains were SIT289, SIT134, and SIT3411, comprising 16.7\% (28/168), 7.14\% (12/168), and 4.76\% (8/168) of the isolates, respectively. Euro-American (51.2\%), EastAfrican-Indian (34.5\%), and M. africanum (9.52\%) were the major lineages identified. Two strains of M. bovis were isolated from TB lymphadenitis cases. The high percentage of clustered strains of $M$. tuberculosis could suggest that a small number of lineages of $M$. tuberculosis are causing the disease in the area while isolation of M. bovis could suggest its zoonotic potential. Additionally, identification of $M$. africanum requires further confirmation by tools with a better discriminatory power.
\end{abstract}

\section{Introduction}

The incidence of TB has continued to increase in many parts of the world [1]. An estimated 9 million people developed TB in 2013, which is $6 \%$ greater than that reported in 2012. A quarter of these cases occurred in the African region [1]. About 1.5 million deaths were attributed to TB globally, of which approximately $75 \%$ occurred in Africa and Southeast Asia [1]. The 22 high TB burden countries (HBCs) collectively accounted for $82 \%$ of all the estimated TB incidences worldwide [1]. Ethiopia is among the top ten HBCs with an estimated incidence rate of 224 patients per 100,000 population [1], which is much greater than $126 / 100,000$ reported globally in 2013 [1]. Moreover, TB is the second most common cause of hospital deaths in the country [2]. TB lymphadenitis in cervical lymph nodes is also common in Ethiopia and accounted for $33 \%$ of all new TB cases [3], which is greater than the global average of 15\% [4]. According to the 2013 Heal TB Project Report of 2014, the incidence of $\mathrm{TB}$ in the Amhara Region (present study area) for one year (October 2012 to September 2013) was estimated to be 247 per 100,000 population (unpublished, URL: http://pdf.usaid.gov/ pdf\%20docs/pa00jn8p.pdf), which is higher than the national incidence rate of Ethiopia during the same year. However, little or no information is available on the type of MTBC species and strains causing the disease in the study area.

Thus, identification of strains circulating in a certain geographic region using molecular tools can contribute to 
the TB control program of that region. Various molecular epidemiology methods allow identifying mycobacterial strains and tracking the transmission of TB in different geographical regions [5]. For example, spoligotyping is widely used for identification of M. tuberculosis [6]. Hence, the purpose of this study was to identify the MTBC isolates to the species and SIT level by the use of spoligotyping.

\section{Materials and Methods}

2.1. Study Area. The study was conducted in Bahir Dar city and its surrounding districts. Bahir Dar is a city in the highland in northwest of Ethiopia and is the capital city of the Amhara Regional Administrative State. The city is located at geographical coordinates of $11^{\circ} 38^{\prime}$ north in latitude and $37^{\circ} 15^{\prime}$ east in longitude. It has an elevation of 1830 meters above sea level and is characterized by hot and humid weather with an average temperature of $29^{\circ} \mathrm{C}$. The population size of the city and its surrounding is 221,991 , of which $180,174(81.2 \%)$ are residing in the Bahir Dar city [7]. To seek a better economic situation, rural inhabitants, particularly those living in a radius of $60 \mathrm{~km}$ around the city, recently migrated and caused the rapid rise in population in Bahir Dar city [8]. The migration led to homelessness and poverty, as the anticipated job opportunities were not realized. In addition, crowded living conditions, lack of ventilation in temporary housing, malnutrition, and lack of education facilitated the spread of TB [9].

2.2. Study Subjects. Smear positive pulmonary TB (PTB) and extrapulmonary TB (EPTB) patients, who were diagnosed as TB cases between September 2012 and January 2014 at Felegehiwot Referral and GAMBY General Hospitals, were included in this study. High TB patient flow, existence of better diagnostic facilities, and skilled human resource were the major reasons for selecting the specified health facilities. The average TB case flow in these two study hospitals over four years (2010-2013) was 321 as assessed from the respective hospital records. $\mathrm{TB}$ patients who visited these health facilities during the study period were enrolled in the study, excluding those below 18 years of age and those who had started treatment prior to launching the study. Children under the age of 18 years were not included as (1) the study was not a pure epidemiological study and its main objective was the identification of strains circulating in the study area, (2) it was not easy to obtain consent from a family member or a guardian for children below 18 years of age, (3) collection of sputum samples can be difficult in children, and (4) systematic differences of MTBC strains comparing the adult population with children were not expected.

2.3. Sample and Data Collection. A structured questionnaire was used to collect data from all study subjects. These data included patient origin, age, sex, household size, TB category, clinical presentation, and family history of $\mathrm{TB}$ infections. Clinical examination of patients suspected to be infected with TB was performed by the attending physicians. Sputum samples submitted for the routine Ziehl Neelsen staining for diagnostic purpose were used for bacteriological examination. Similarly, fine needle aspirates (FNA) collected by a pathologist for the routine diagnosis of TB were used for mycobacterial culture.

2.4. Mycobacterium Culture. Isolation of mycobacteria was made on Lowenstein-Jensen (LJ) medium using the procedure described by the National TB and Leprosy Control Programme Guideline [10] that was adopted from WHO guideline [11]. Both sputum and FNA samples were cultured at the Bahir Dar Regional Health Research Laboratory Centre. Briefly, sputum or FNA samples were homogenized and decontaminated with an equal volume of $4 \% \mathrm{NaOH}$ by centrifugation at $3000 \mathrm{rpm}$ for 15 minutes at room temperature. The supernatant was decanted while the sediment was neutralized with $2 \mathrm{~N} \mathrm{HCl}$ using phenol red as an indicator. Neutralization was achieved when the color of the solution changed from purple to yellow. About $100 \mu \mathrm{L}$ of the suspension was inoculated on four sterile LJ medium slopes (two were supplemented with pyruvate and the other two with glycerol) and then incubated at $37^{\circ} \mathrm{C}$ with weekly examination for growth. Specimens without colonies at eighth week after culturing were considered as negative. Specimens with growth of colonies were examined by Ziehl Neelsen microscopy. AFB positive colonies were harvested and resuspended in $200 \mu \mathrm{L}$ sterile distilled water. Thereafter, they were inactivated by heating at $80^{\circ} \mathrm{C}$ for 45 minutes in a water bath and transported to Aklilu Lemma Institute of Pathobiology (ALIPB), Addis Ababa University, for spoligotyping.

2.5. Spoligotyping. All of the 168 isolates were characterized by spoligotyping as previously described by Kamerbeek et al. [12] following the instruction supplied with the spoligotyping kit (Ocimum Biosolutions Company, Ijsselstein, The Netherlands). Briefly, the direct repeat (DR) region of the isolate was amplified using DRa and DRb primers. The amplified biotinylated products were hybridized with a set of 43 oligonucleotides covalently bound to a membrane (Animal and Plant Health Agency, Great Britain). Known strains of $M$. bovis and M. tuberculosis $\mathrm{H} 37 \mathrm{Rv}$ were used as positive controls, whereas Qiagen water (Qiagen Company, Germany) was used as a negative control. Hybridized DNA was detected by the enhanced chemiluminescence method. Images were captured by exposure to X-ray film (Hyperfilm ECL, Amersham) as specified by the manufacturer's instruction. The presence and absence of spacers were visualized on film as black and white squares, respectively.

2.6. Comparison of Experimental Data with the SITVIT2 Database. The spoligotype patterns were converted into binary and octal formats and entered into the open source spoligotype database available at the website http://www.pasteur-guadeloupe.fr:8081/SITVIT_ONLINE/tools.jsp. The shared international spoligotype (SIT) number and lineages/ sublineages were retrieved from the database. The results were compared with the existing designations in the SITVIT2 database of Institute Pasteur de la Guadeloupe. Two or more mycobacterial isolates sharing a spoligotype pattern in the study were identified as a cluster, whereas single spoligotype patterns in the study were recognized as unique. Strains matching a preexisting pattern in the SITVIT2 database were 
identified with the SIT number, whereas strains for which SIT numbers were not found from the database were considered as orphan strains. In addition, the online tool "Run TBLineage" (http://tbinsight.cs.rpi.edu/run_tb_lineage.html) was also used to predict the major lineages to which the strains belong by a conformal Bayesian network (CBN) analysis.

2.7. Statistical Analysis. The statistical analysis was performed using STATA software version 12 [13]. Descriptive statistics were used to depict the demographic and clinical variables. Chi-square or Fisher's exact tests were used to evaluate the association of clusters and major lineages with selected patient characteristics. $P$ values of less than 0.05 were considered statistically significant.

2.8. Ethical Considerations. Ethical clearance was obtained from Ethical Review Board (Ref. number IRB/05-02/2013) of the Aklilu Lemma Institute of Pathobiology, Addis Ababa University. In addition, permission was obtained from the Research Committee of Bureau of Health, Amhara National Regional State, Ethiopia.

\section{Results}

3.1. Demographic and Clinical Characteristics of the Study Subjects. Data generated from 168 subjects were used in the analysis of the demographic and clinical results. Among the study participants, $52.4 \%$ were female, $73.8 \%$ were in age range of $18-39$ years, $84.5 \%$ were new cases, $27.4 \%$ had a history of TB pertaining to one of their family members, and $67.9 \%$ were EPTB patients. Surprisingly, all EPTB cases were identified as TB lymphadenitis (TBLN), of which $67(60.4 \%)$ and 18 (16.2\%) were TBLN in cervical and axillary lymph nodes, respectively. Of the 168 isolates, $33.9 \%$ and $25.6 \%$ originated from South Gondar and West Gojjam, respectively (Table 1). Nonetheless, the sociodemographic and clinical characteristics of the patients did not affect the clustering rates and distribution of the lineages of MTBC strains (Table 1).

3.2. Spoligotyping Patterns of Mycobacterium tuberculosis Complex Strains. A total of 168 MTBC isolates were spoligotyped, and 89 (53\%) different spoligotype patterns (strains) were identified. Clustering of isolates into strains was observed, and a total of 98 isolates were grouped in 19 (58.3\%) different clusters of strains. The dominant strains were SIT289, SIT134, and SIT3411, each consisting of 28 (16.7\%), $12(7.14 \%)$, and $8(4.76 \%)$ isolates, respectively. These strains contributed $28.6 \%(48 / 168)$ of all isolates with known spoligotype patterns. Out of the 89 spoligotype patterns (strains), 44 strains associated with 122 isolates matched the preexisting patterns in the SITVIT2 database while the remaining 45 spoligotype patterns associated with 46 isolates were not registered in the international spoligotype SITVIT2 database and thus designated as orphan strains.

Classification of MTBC strains showed the occurrence of the following lineages: Euro-American $(86 / 168 ; 51.2 \%)$, East-African Indian (58/168; 34.5\%), M. africanum (16/168; $9.52 \%)$, Indo-Oceanic $(6 / 168 ; 3.57 \%)$, and $M$. bovis $(2 / 168$;

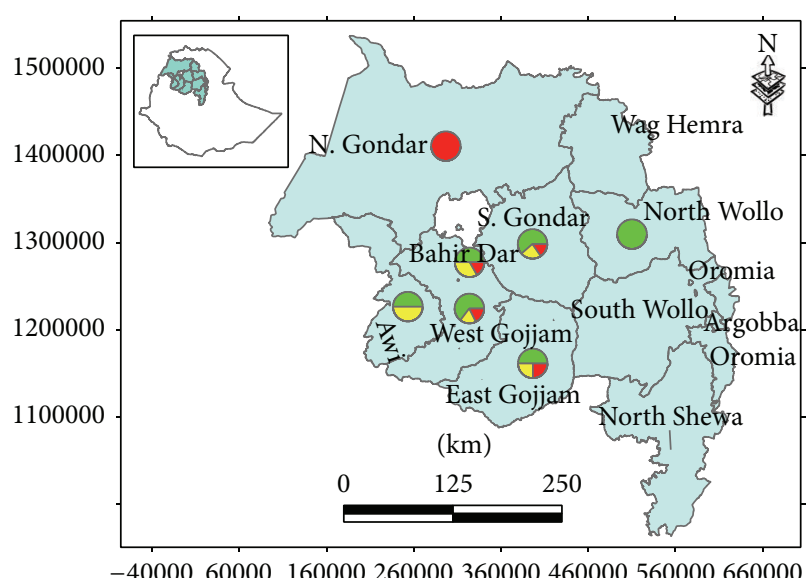

289 (CAS1-Delhi) $134(\mathrm{H} 3)$

FIGURE 1: Map showing the distribution of the three dominant strains in Bahir Dar city and its surrounding zones, northwest Ethiopia. The green coloured portion of the icons in the map shows SIT289 with frequency of $14 \%$ (8/57) for South Gondar, 8.33\% (2/24) for East Gojjam, 25.9\% (11/43) for West Gojjam, 15\% (3/20) for Bahir Dar Special Zone, and 15\% (3/20) for Awi Zone. The yellow colour shows SIT134 with frequency of 5.26\% (3/57) for South Gondar, $4.17 \%(1 / 24)$ for East Gojjam, 6.98\% (3/43) for West Gojjam, 10\% $(2 / 20)$ for Bahir Dar Special Zone, and 15\% (3/20) for Awi Zone. The red colour shows SIT3411 with frequency of $50 \%(1 / 2)$ for North Gondar, 3.51\% (2/57) for South Gondar, 4.17\% (1/24) for East Gojjam, 6.98\% (3/43) for West Gojjam, and 5\% (1/20) for Bahir Dar Special Zone.

1.19\%). Two of the spoligotypes (i.e., SIT982 and SIT665) were M. bovis and both were isolated from EPTB cases. Detailed spoligotyping results and their corresponding SITs/orphan strains and lineages are summarized in Tables 2 and 3.

3.3. Distribution of Strains and Lineages in the Study Area. The majority of MTBC strains were identified from the South Gondar Zone (57/168; 33.9\%) followed by the West Gojjam Zone $(43 / 168 ; 25.6 \%)$, each with a strain-clustering rate of 17.3\% (Table 1). The distribution of the three dominant strains (SIT289, SIT134, and SIT3411) in the area is depicted in Figure 1.

The Euro-American (EA), East-African-Indian (EAI), Indo-Oceanic (IO), and M. africanum (MA) lineages were identified in all study zones, whereas $M$. bovis (MB) was recorded only from patients with TBLN located in South Gondar. Figure 2 depicts the distribution of the major lineages.

\section{Discussion}

In the present study, MTBC species were isolated from 168 TB patients from Bahir Dar city itself and the surrounding districts who visited health institutions in Bahir Dar city. The isolates were identified at strain and lineage levels on the basis of spoligotyping. Identification at a higher level of resolution 
TABLE 1: Demographic and clinical characteristics of the study subjects and their association with spoligotype clustering and major lineages $(n=168)$.

\begin{tabular}{|c|c|c|c|c|c|c|c|c|c|c|c|}
\hline \multirow{2}{*}{ Characteristics } & \multirow{2}{*}{ Number of isolates, $n(\%)$} & \multicolumn{4}{|c|}{ Number of isolates clustered versus unique ones } & \multicolumn{6}{|c|}{ Major lineages by CBN } \\
\hline & & Clustered & Unique & Clustering rate & $P$ value & EA & EAI & $\mathrm{IO}$ & MA & $\mathrm{MB}$ & $P$ value \\
\hline \multicolumn{12}{|l|}{ Patient origin (zonal) } \\
\hline North Gondar & $2(1.19)$ & 1 & 1 & 0.6 & \multirow{7}{*}{0.502} & 1 & 1 & 0 & 0 & 0 & \multirow{7}{*}{0.822} \\
\hline South Gondar & $57(33.9)$ & 29 & 28 & 17.3 & & 27 & 18 & 3 & 7 & 2 & \\
\hline East Gojjam & $24(14.3)$ & 12 & 12 & 7.14 & & 13 & 9 & 1 & 1 & 0 & \\
\hline West Gojjam & $43(25.6)$ & 29 & 14 & 17.3 & & 19 & 18 & 0 & 6 & 0 & \\
\hline Bahir Dar Special & 20 (11.9) & 13 & 7 & 7.74 & & 15 & 4 & 0 & 1 & 0 & \\
\hline Awi & $20(11.9)$ & 12 & 8 & 7.14 & & 10 & 7 & 2 & 1 & 0 & \\
\hline North Wollo & $2(1.19)$ & 2 & 0 & 1.19 & & 1 & 1 & 0 & 0 & 0 & \\
\hline \multicolumn{12}{|l|}{ Age (years) } \\
\hline $18-28$ & $83(49.4)$ & 47 & 36 & 28 & \multirow{4}{*}{0.181} & 43 & 30 & 0 & 10 & 0 & \multirow{4}{*}{0.277} \\
\hline $29-39$ & $41(24.4)$ & 28 & 13 & 16.7 & & 19 & 16 & 2 & 3 & 1 & \\
\hline $40-50$ & 20 (11.9) & 13 & 7 & 7.74 & & 13 & 4 & 2 & 1 & 0 & \\
\hline$>50$ & $24(14.3)$ & 10 & 14 & 5.95 & & 11 & 8 & 2 & 2 & 1 & \\
\hline \multicolumn{12}{|l|}{ Sex } \\
\hline Male & $80(47.6)$ & 51 & 29 & 30.4 & \multirow{2}{*}{0.175} & 41 & 27 & 3 & 9 & 0 & \multirow{2}{*}{0.674} \\
\hline Female & $88(52.4)$ & 47 & 41 & 28 & & 45 & 31 & 3 & 7 & 2 & \\
\hline \multicolumn{12}{|l|}{ Household size } \\
\hline$\leq 4$ & $85(50.6)$ & 49 & 36 & 29.2 & \multirow{2}{*}{0.855} & 42 & 31 & 2 & 9 & 1 & \multirow{2}{*}{0.876} \\
\hline$>4$ & $83(49.4)$ & 49 & 34 & 29.2 & & 44 & 27 & 4 & 7 & 1 & \\
\hline \multicolumn{12}{|l|}{ TB category } \\
\hline Retreatment & $26(15.5)$ & 15 & 11 & 8.93 & \multirow{2}{*}{0.943} & 15 & 8 & 1 & 2 & 0 & \multirow{2}{*}{0.93} \\
\hline New & $142(84.5)$ & 83 & 59 & 49.4 & & 71 & 50 & 5 & 14 & 2 & \\
\hline \multicolumn{12}{|l|}{ Family TB history } \\
\hline Yes & $46(27.4)$ & 30 & 16 & 17.9 & \multirow{2}{*}{0.266} & 26 & 14 & 1 & 5 & 0 & \multirow{2}{*}{0.758} \\
\hline No & $122(72.6)$ & 68 & 54 & 40.5 & & 60 & 44 & 5 & 11 & 2 & \\
\hline \multicolumn{12}{|l|}{ Clinical presentation } \\
\hline ЕРТВ & $114(67.9)$ & 67 & 47 & 39.9 & \multirow{3}{*}{0.867} & 53 & 43 & 6 & 10 & 2 & \multirow{3}{*}{0.16} \\
\hline РTB & $54(32.1)$ & 31 & 23 & 18.5 & & 33 & 15 & 0 & 6 & 0 & \\
\hline Total & $168(100)$ & 98 & 70 & 58.3 & & 88 & 58 & 6 & 16 & 2 & \\
\hline
\end{tabular}

EA: Euro-American; EAI: East-African Indian; IO: Indo-Oceanic; MA: M. africanum; MB: M. bovis; CBN: conformal Bayesian network; $P$ values were presented at $95 \%$ confidence interval and $P<0.05$ considered statistically significant.

by using variable nucleotide tandem repeat (VNTR) typing, facilitated by mycobacterial interspersed repetitive units(MIRU-) VNTR, is desirable although this method is not currently available in Ethiopia.

Spoligotyping of 168 mycobacterial isolates revealed 89 distinct patterns, which corresponded to $53 \%$ of genotype diversity. The high diversity of spoligotypes strains that we observed in this study was consistent with the 59\% reported by Tessema et al. [14] but higher than the percentages reported earlier by other studies in Ethiopia [3, 15-18]. This finding suggests the circulation of genetically variable strains in the study area, which could be the result of significant migration of infected people to Bahir Dar city and its surroundings from other regions of the country. In addition, the long period of MTBC clonal evolution may contribute to the diversity of strains [19]. Ninety-eight mycobacterial isolates were grouped into 19 clusters with an overall clustering percentage of $58.3 \%$. The clustering rate observed in this study was slightly higher than those reported previously in Ethiopia $[14,18,20]$. On the other hand, it was lower than those reported by several other national studies [15, 16, 21] and international studies (e.g., South Africa [22] and Malawi [23]). The observed differences in clustering rates might be related to differences of sociocultural origin, sanitation, and population density. High level of strain clustering could suggest recent and ongoing TB transmission [24].

The prevalent strains identified in this study were SIT289, SIT134, and SIT3411. All three strains seem to be specific for the Bahir Dar city and its surroundings since they were not reported previously from other sites in Ethiopia [3, $14,17,18]$. However, in the SITVIT database, SIT289 has only been reported from Brazil and Europe (mainly France, French Guiana, Martinique, Italy, Netherlands, and Sweden), while SIT134 has been reported from Central Asia and Middle East (Bangladesh, India, Pakistan, and Saudi Arabia), Australia, Netherlands, and United States of America [25]. 
TABLE 2: Spoligotype patterns of 44 shared types and their corresponding lineages/sublineages identified from a total of $168 \mathrm{Mycobacterium}$ tuberculosis complex strains isolated in the Bahir Dar region.

\begin{tabular}{|c|c|c|c|c|c|}
\hline SIT & $\begin{array}{c}\text { Isolates with } \\
\text { similar pattern }\end{array}$ & $\begin{array}{c}\text { SITVIT2 } \\
\text { lineage/sublineage }\end{array}$ & $\mathrm{CBN}^{*}$ lineage & Octal number & Binary format \\
\hline 20 & 1 & LAM1 & EA & 677777607760771 & | \\
\hline 35 & 4 & Ural-1 & EA & 777737777420771 & " \\
\hline 37 & 1 & $\mathrm{~T} 3$ & EA & 777737777760771 & 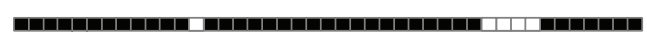 \\
\hline 41 & 3 & Turkey & EA & 777777404760771 & " \\
\hline 50 & 3 & $\mathrm{H} 3$ & EA & 777777777720771 & 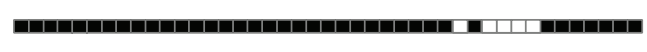 \\
\hline 51 & 1 & $\mathrm{~T}$ & EA & 777777777760700 & 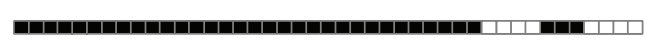 \\
\hline 52 & 1 & $\mathrm{~T} 2$ & EA & 777777777760731 & Manm \\
\hline 53 & 6 & $\mathrm{~T}$ & EA & 777777777760771 & " \\
\hline 54 & 3 & Manu2 & EA & 777777777763771 & | \\
\hline 93 & 1 & LAM5 & EA & 777737607760771 & 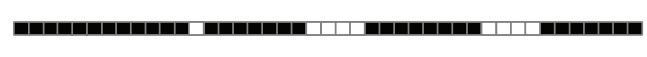 \\
\hline 134 & 12 & $\mathrm{H} 3$ & EA & 777777777720631 & 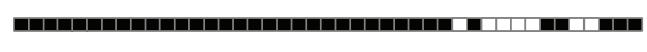 \\
\hline 137 & 2 & $\mathrm{X} 2$ & EA & 777776777760601 & 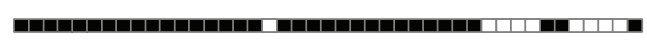 \\
\hline 149 & 5 & T3-ETH & EA & 777000377760771 & 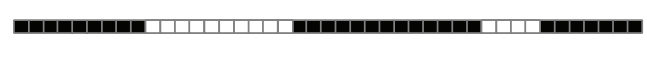 \\
\hline 168 & 3 & $\mathrm{H} 3$ & EA & 777777777720671 & 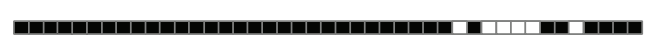 \\
\hline 205 & 1 & $\mathrm{~T}$ & EA & 737777777760771 & 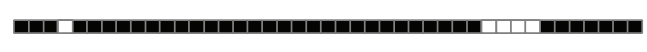 \\
\hline 336 & 1 & $\mathrm{X} 1$ & EA & 777776777760731 & 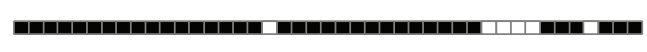 \\
\hline 699 & 1 & H3 & EA & 677777777720571 & 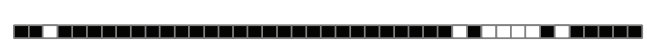 \\
\hline 777 & 1 & Ural-1 & EA & 777777777420771 & " \\
\hline 817 & 1 & Ural-1 & EA & 777777777420731 & 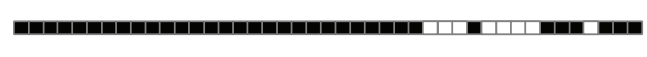 \\
\hline 1166 & 1 & $\mathrm{~T}$ & EA & 777377777760771 & 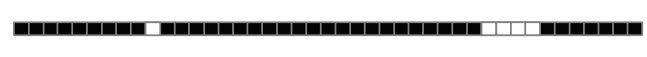 \\
\hline 1552 & 1 & $\mathrm{H} 1$ & EA & 777777774020631 & 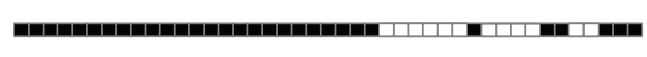 \\
\hline 1688 & 1 & $\mathrm{~T}$ & EA & 777777403760771 & " \\
\hline 1877 & 1 & $\mathrm{~T}$ & EA & 737377777760771 & | \\
\hline 2007 & 1 & $\mathrm{~T} 3$ & EA & 777737677760771 & | \\
\hline 2409 & 1 & $\mathrm{~T} 3$ & EA & 777737757760771 & 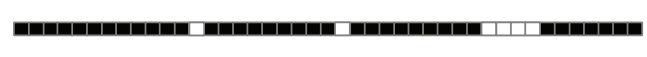 \\
\hline 3134 & 1 & $\mathrm{H} 3$ & EA & 777737377720771 & " \\
\hline 3411 & 8 & T3-ETH & EA & 777002377760771 & 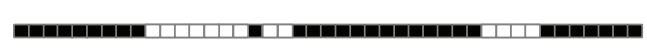 \\
\hline 3412 & 2 & $\mathrm{~T} 4$ & EA & 777003377760771 & 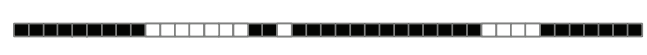 \\
\hline 21 & 2 & CAS1-Kili & EAI & 703377400001771 & | \\
\hline 25 & 7 & CAS1-Delhi & EAI & 703777740003171 & 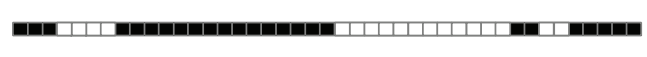 \\
\hline 26 & 2 & CAS1-Delhi & EAI & 703777740003771 & 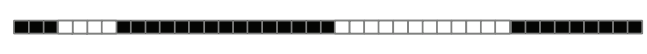 \\
\hline 289 & 28 & CAS1-Delhi & EAI & 703777740003571 & 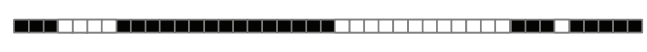 \\
\hline 754 & 1 & CAS1-Delhi & EAI & 503777740003771 & 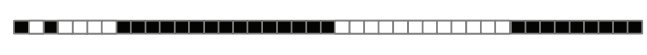 \\
\hline 952 & 2 & CAS1-Delhi & EAI & 603777740003771 & 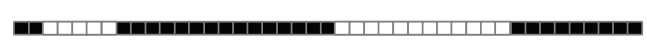 \\
\hline 1200 & 2 & Unknown & EAI & 703777747777771 & 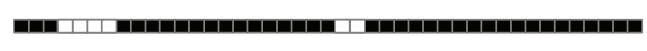 \\
\hline 1551 & 1 & CAS1-Delhi & EAI & 701777740003771 & 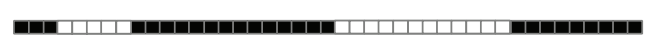 \\
\hline 2359 & 2 & CAS1-Delhi & EAI & 703677740003171 & 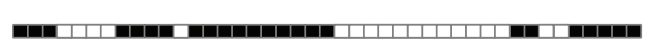 \\
\hline 343 & 1 & Unknown & M. africanum & 700000007175771 & 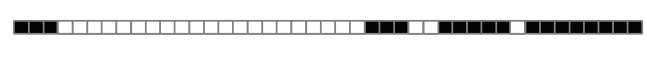 \\
\hline 910 & 1 & Unknown & M. africanum & 700000007177771 & 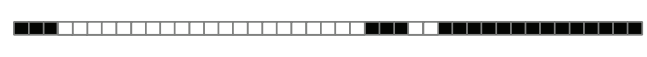 \\
\hline 1729 & 1 & Unknown & M. africanum & 700000004177771 & 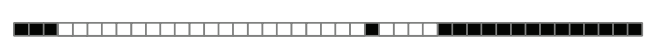 \\
\hline 3409 & 1 & AFRI & M. africanum & 700020047177771 & 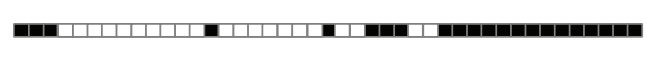 \\
\hline 665 & 1 & BOV_1 & M. bovis & 616773777777600 & 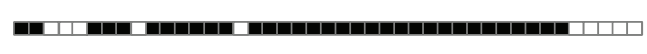 \\
\hline 982 & 1 & $\mathrm{BOV}$ & M. bovis & 416773777777600 & 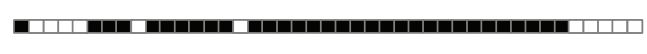 \\
\hline 523 & 1 & Manu_ancest & $\mathrm{IO}$ & 777777777777771 & 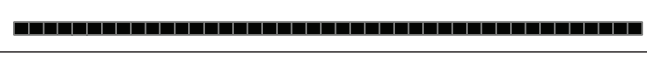 \\
\hline
\end{tabular}

${ }^{*} \mathrm{CBN}$ : conformal Bayesian network; unknown: designates patterns with signatures that do not belong to any of the major lineages/sublineages described in the SITVIT2 database. The 168 isolates were grouped into 89 different spoligotype patterns (strains). Of the total 89 strains, 44 strains (patterns) have already been registered in the SITVIT2 database (Table 2), while the remaining 45 patters were orphans and presented in Table 3. The dominant strains were SIT289 (28 isolates), SIT134 (12 isolates), and SIT3411 (8 isolates) (Table 2). Furthermore, the 168 isolates were grouped into five different lineages including the EuroAmerican, East-African Indian, M. africanum, Indo-Oceanic, and M. bovis lineages in the order of decreasing percentage. 
TABLE 3: Spoligotype patterns of 45 orphan strains and their corresponding lineages/sublineages identified from a total of $168 \mathrm{Mycobacterium}$ tuberculosis complex isolates collected in tuberculosis patients in the Bahir Dar region.

\begin{tabular}{|c|c|c|c|c|c|}
\hline SIT & $\begin{array}{c}\text { Isolates with } \\
\text { similar pattern }\end{array}$ & $\begin{array}{c}\text { SITVIT2 } \\
\text { lineage/sublineage }\end{array}$ & $\mathrm{CBN}^{*}$ lineage & Octal number & Binary format \\
\hline Orphan & 1 & $\mathrm{~T}$ & EA & 776603777760771 & | \\
\hline Orphan & 1 & $\mathrm{~T}$ & EA & 777737347760771 & " \\
\hline Orphan & 1 & $\mathrm{~T}$ & EA & 276777777760771 & 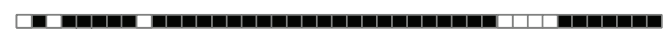 \\
\hline Orphan & 1 & T3-ETH & EA & 777002377420771 & manmon \\
\hline Orphan & 1 & $\mathrm{~T} 2$ & EA & 777777403760731 & " \\
\hline Orphan & 1 & EAI & EA & 777760370000000 & 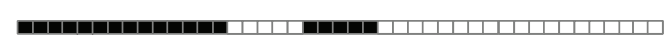 \\
\hline Orphan & 1 & EAI & EA & 777770370000000 & 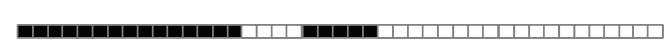 \\
\hline Orphan & 1 & T3-ETH & EA & 777002377760731 & m \\
\hline Orphan & 1 & T1-RUS2 & EA & 770002001760771 & Em \\
\hline Orphan & 1 & EAI & EA & 777760370000000 & 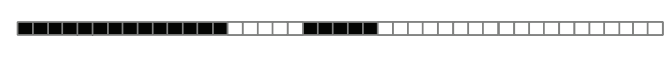 \\
\hline Orphan & 1 & $\mathrm{~T}$ & EA & 276777737760771 & C" \\
\hline Orphan & 1 & $\mathrm{~T}-\mathrm{H} 37 \mathrm{Rv}$ & EA & 777777444760771 & m " \\
\hline Orphan & 1 & $\mathrm{X} 1$ & EA & 400002757760771 & m \\
\hline Orphan & 1 & $\mathrm{~T} 2$ & EA & 777777403760731 & " \\
\hline Orphan & 1 & Manu2 & EA & 577747777767771 & 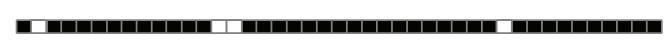 \\
\hline Orphan & 1 & $\mathrm{H} 3$ & EA & 777737377720731 & m " \\
\hline Orphan & 1 & Manu2 & EA & 777777774363771 & 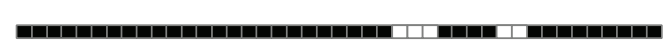 \\
\hline Orphan & 1 & LAM3 & EA & 760002007760771 & m \\
\hline Orphan & 1 & AFRI & EAI & 700022044037771 & Bum \\
\hline Orphan & 1 & Unknown & EAI & 000022000003771 & 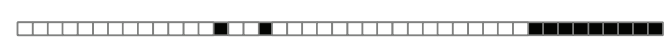 \\
\hline Orphan & 1 & CAS & EAI & 700002000000771 & 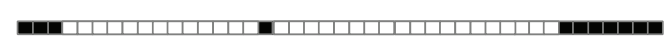 \\
\hline Orphan & 1 & PINI2 & EAI & 400200000000751 & m \\
\hline Orphan & 1 & $\mathrm{H} 2$ & EAI & 700000004037771 & 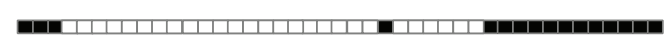 \\
\hline Orphan & 1 & CAS1-Delhi & EAI & 703602040003571 & 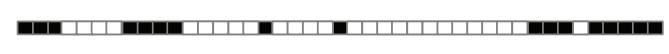 \\
\hline Orphan & 1 & PINI2 & EAI & 000022000003771 & 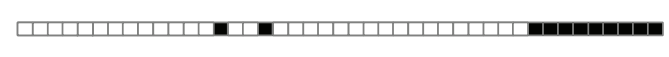 \\
\hline Orphan & 1 & $\mathrm{H}$ & EAI & 000002004020631 & 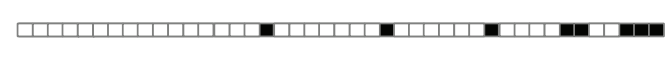 \\
\hline Orphan & 1 & CAS1-Delhi & EAI & 703677740003571 & 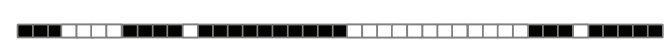 \\
\hline Orphan & 1 & CAS & EAI & 703777700001171 & 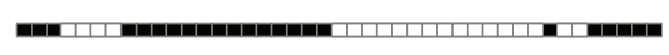 \\
\hline Orphan & 1 & CAS1-Delhi & EAI & 703622040003571 & 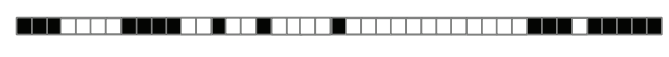 \\
\hline Orphan & 1 & AFRI & M. africanum & 700022007177771 & 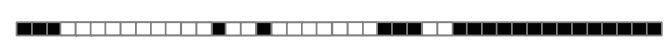 \\
\hline Orphan & 1 & AFRI & M. africanum & 700002044177771 & m \\
\hline Orphan & 1 & AFRI & M. africanum & 700020044177771 & m \\
\hline Orphan & 2 & AFRI & M. africanum & 700002004177771 & | \\
\hline Orphan & 1 & AFRI & M. africanum & 700002004177771 & | \\
\hline Orphan & 1 & AFRI & M. africanum & 771022044177771 & 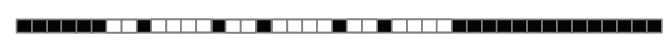 \\
\hline Orphan & 1 & AFRI & M. africanum & 700002004177771 & mane \\
\hline Orphan & 1 & AFRI & M. africanum & 700002007177771 & 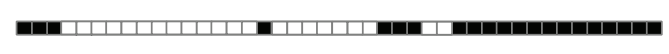 \\
\hline Orphan & 1 & AFRI & M. africanum & 700002007177771 & 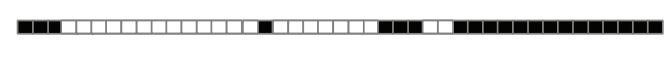 \\
\hline Orphan & 1 & AFRI & M. africanum & 700022004177771 & 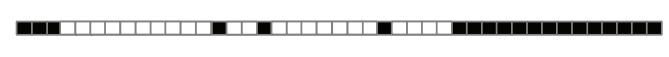 \\
\hline Orphan & 1 & AFRI & M. africanum & 700022047177771 & 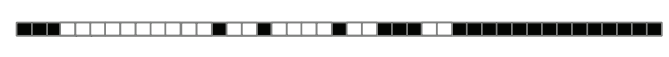 \\
\hline Orphan & 1 & Manul & IO & 773777747777771 & 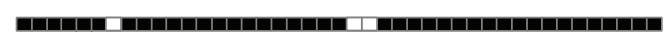 \\
\hline Orphan & 1 & Manu2 & IO & 773777744203771 & 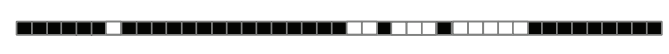 \\
\hline Orphan & 1 & Manul & IO & 753777747777771 & 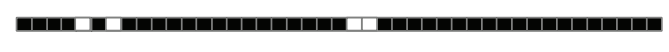 \\
\hline Orphan & 1 & Manul & IO & 717777777777771 & ma \\
\hline Orphan & 1 & EAI & IO & 717777776003771 & | \\
\hline
\end{tabular}

${ }^{*} \mathrm{CBN}$ : conformal Bayesian network; unknown: designates patterns with signatures that do not belong to any of the major lineages/sublineages described in the SITVIT2 database. Forty-five of the total 89 strains were identified as orphan strains in the present study and shown in Table 3 . The orphan strains belonged to four lineages including Euro-American, East-African Indian, M. africanum, and Indo-Oceanic lineages. 

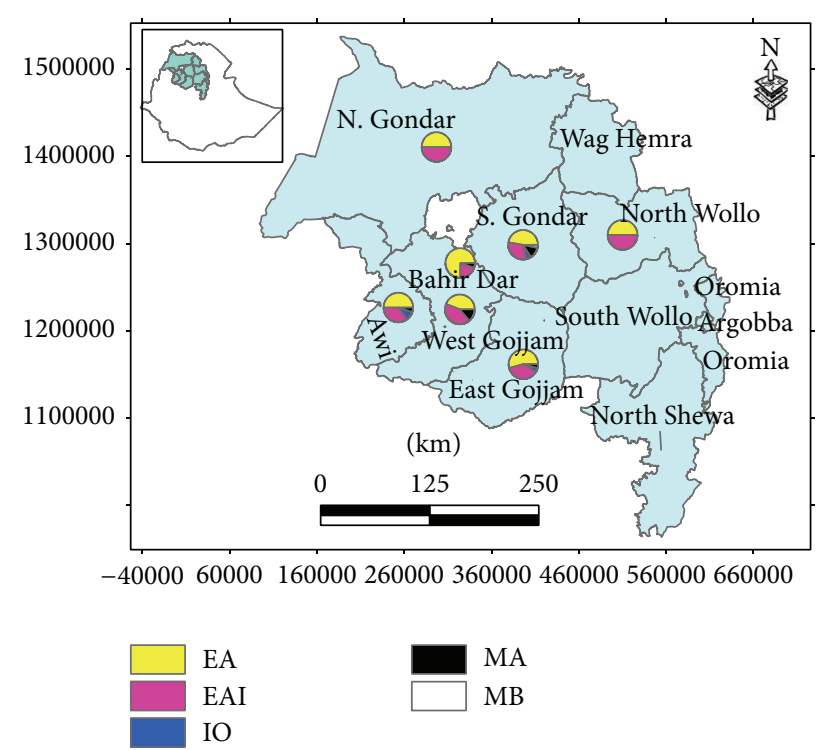

FIGURE 2: Distribution of mycobacterial lineages in the different zones of the Amhara Region, northwest Ethiopia. Five different lineages were identified. The Euro-American (EA) lineage is represented by yellow segments with a frequency of $50 \%(1 / 2)$ for North Gondar, 47.4\% (27/57) for South Gondar, 54.2\% (13/24) for East Gojjam, 44.2\% (19/43) for West Gojjam, 75\% (15/20) for Bahir Dar Special Zone, 50\% (10/20) for Awi, and 50\% (1/2) for North Wollo zone. The East-African Indian (EAI) lineage is represented by red segments with a frequency of $50 \%(1 / 2)$ for North Gondar, $31.6 \%$ (18/57) for South Gondar, 37.5\% (9/24) for East Gojjam, 41.9\% (18/43) for West Gojjam, 20\% (4/20) for Bahir Dar Special Zone, $35 \%(2 / 20)$ for Awi, and $50 \%$ (1/2) for North Wollo zone. The IndoOceanic (IO) lineage is shown by blue segments with the frequency of 5.26\% (3/57) for South Gondar, 4.17\% (1/24) for East Gojjam, and $10 \%(2 / 20)$ for Awi Zone. The M. africanum (MA) lineage is represented by black segments with a frequency of $12.3 \%(7 / 57)$ for South Gondar, 4.17\% (1/24) for East Gojjam, 14\% (6/43) for West Gojjam, and 5\% (1/20) each for Bahir Dar Special and Awi Zones. $M$. bovis (MB) was identified only in patients with TB lymphadenitis (TBLN) located in South Gondar with the prevalence of 3.51\% (2/57) showed by a white coloured segment.

A considerable number (46/168) of orphan strains were also recorded in this study. This is nearly identical to the average reported by Tessema et al. [14], Belay et al. [15], and Mihret et al. [16] in Ethiopia. The existence of mixed infections may also complicate spoligotyping results $[6,26]$, and hence higher resolution molecular tools should be applied toreveal thus far undefined mixed spoligotyping signatures.

Five different major lineages, namely, Euro-American, East-African-Indian, Indo-Oceanic, $M$. africanum, and $M$. bovis, were identified in this study. Euro-American was the dominant lineage, and more than half $(51.5 \%)$ of the overall strains belonged to this lineage. This finding agreed with the results of previous studies in Ethiopia [3, 16-18] and Morocco [27]. The high proportion of new MTBC lineages is supposed to be related to their successful geographical spread as compared to ancient lineages [28,29]. Even though Euro-American was identified as the prevalent major lineage, the CAS1_DELHI sublineage (consisting of 46 isolates) in the East-African Indian lineage appeared to have had a high transmission rate in our study population. This lineage is localized in South Asia, preferentially India, countries of the Middle East, and several other regions, including Africa [6]. It can be hypothesized that East-African Indian ancestral strains spread back from Asia to Africa through India as a result of human migration [30].

Screening of the SITVIT2 database also identified 9.52\% $(16 / 168)$ of the isolates as members of M. africanum. The clustering rate was $1.19 \%(2 / 168)$, indicative of a low rate of recent human-to-human transmission. Since it was not reported previously in Ethiopia [3, 14-18], isolation and identification of $M$. africanum in this study represent a novel finding. Further studies are needed to explore evolutionary aspects that may have contributed to the spread of $M$. africanum in the study population. Two strains of M. bovis (SIT982 and SIT665) were identified in this study. This finding was interesting and could implicate the public health importance of $M$. bovis in northwestern Ethiopia.

\section{Conclusions}

Molecular characterization of MTBC isolates from TB patients in Bahir Dar city and its surroundings was performed using spoligotyping. The high percentage of clustered strains of $M$. tuberculosis could suggest that a small number of lineages of $M$. tuberculosis are causing the disease in the area and isolation of $M$. bovis could suggest its zoonotic potential in the study area. Meanwhile, identification of $M$. africanum requires confirmation by molecular tools with a better discriminatory power than spoligotyping.

\section{Conflict of Interests}

The authors declared that they have no competing interests.

\section{Authors' Contribution}

Anwar Nuru was involved in the design, data collection and laboratory work, and statistical analysis and interpretation of data and drafted the paper; Gezahegne Mamo was involved in the design, interpretation, and critical revision of the paper; Adane Worku was involved in the spoligotyping of the isolates and interpretation; Aschalew Admasu was involved in data collection, TB culture, and interpretation; Girmay Medhin was involved in the design, statistical analysis, and critical revision of the paper; Rembert Pieper was involved in critical revision of the paper; Gobena Ameni was involved in the design, interpretation, and critical revision of the paper. All authors have read and approved the paper for submission.

\section{Acknowledgments}

This study was jointly funded by the National Institute of Health (NIH, USA) through its H3Africa Consortium 
Program (Grant Ref. no. U01HG007472-01), Addis Ababa University through its Thematic Research Program, and University of Gondar. The authors are grateful to Dr. Getachew Mulualem and the other technical personnel for collecting samples as well as the institutions which facilitated the conducting of this study. The authors would like to acknowledge David Couvin for comparing the experimental strains with SITVIT2 database and Eyaya Belay for his support in doing the GIS maps.

\section{References}

[1] WHO, Global Tuberculosis Report, WHO, Geneva, Switzerland, 2014.

[2] Federal Ministry of Health, Guidelines for Clinical and Programmatic Management of TB, Leprosy and TB/HIV in Ethiopia, Federal Ministry of Health, Addis Ababa, Ethiopia, 15th edition, 2012.

[3] R. Firdessa, S. Berg, E. Hailu et al., "Mycobacterial lineages causing pulmonary and extrapulmonary Tuberculosis, Ethiopia," Emerging Infectious Diseases, vol. 19, no. 3, pp. 460-463, 2013.

[4] WHO, Global Tuberculosis Report, WHO, Geneva, Switzerland, 2011.

[5] D. García De Viedma, I. Mokrousov, and N. Rastogi, "Innovations in the molecular epidemiology of tuberculosis," Enfermedades Infecciosas y Microbiología Clínica, vol. 29, no. 1, pp. 8-13, 2011.

[6] K. Brudey, J. R. Driscoll, L. Rigouts et al., "Mycobacterium tuberculosis complex genetic diversity: mining the fourth international spoligotyping database (SpolDB4) for classification, population genetics and epidemiology," BMC Microbiology, vol. 6, article 23, 2006.

[7] Central Statistics Agency, Summary and Statistical Report of the 2007 Population and Housing Census: Population Size by Age and Sex, Central Statistics Agency, Addis Ababa, Ethiopia, 2007.

[8] M. Ali, "Status of homeless population in urban Ethiopia: a case study of Amhara region," International Journal of Management and Social Sciences Research, vol. 3, no. 1, pp. 61-68, 2014.

[9] C. W. Schmidt, "Linking TB and the environment: an overlooked mitigation strategy," Environmental Health Perspectives, vol. 116, no. 11, pp. A478-A485, 2008.

[10] Federal Ministry of Health, Tuberculosis, Leprosy and TB/HIV Prevention and Control Programme, Federal Ministry of Health, Addis Ababa, Ethiopia, 4th edition, 2008.

[11] WHO, Laboratory Services in Tuberculosis Control: Culture. Part III, World Health Organization (WHO), Geneva, Switzerland, 1998.

[12] J. Kamerbeek, L. Schouls, A. Kolk et al., "Simultaneous detection and strain differentiation of Mycobacterium tuberculosis for diagnosis and epidemiology," Journal of Clinical Microbiology, vol. 35, no. 4, pp. 907-914, 1997.

[13] StataCorp LP, Stata Statistical Software: Release 12, StataCorp LP, College Station, Tex, USA, 2011.

[14] B. Tessema, J. Beer, M. Merker et al., "Molecular epidemiology and transmission dynamics of Mycobacterium tuberculosis in Northwest Ethiopia: new phylogenetic lineages found in Northwest Ethiopia," BMC Infectious Diseases, vol. 13, no. 1, article 131, 2013.
[15] M. Belay, G. Ameni, G. Bjune, D. Couvin, N. Rastogi, and F. Abebe, "Strain diversity of Mycobacterium tuberculosis isolates from pulmonary tuberculosis patients in afar pastoral region of Ethiopia," BioMed Research International, vol. 2014, Article ID 238532, 12 pages, 2014.

[16] A. Mihret, Y. Bekele, M. Aytenew et al., "Modern lineages of Mycobacterium tuberculosis in Addis Ababa, Ethiopia: implications for the tuberculosis control programe," African Journal of Health Sciences, vol. 12, no. 3, pp. 339-344, 2012.

[17] L. Garedew, A. Mihret, G. Mamo et al., "Strain diversity of mycobacteria isolated from pulmonary tuberculosis patients at Debre Birhan Hospital, Ethiopia," International Journal of Tuberculosis and Lung Disease, vol. 17, no. 8, pp. 1076-1081, 2013.

[18] T. Debebe, A. Admassu, G. Mamo, and G. Ameni, "Molecular characterization of Mycobacterium tuberculosis isolated from pulmonary tuberculosis patients in Felege Hiwot Referral Hospital, northwest Ethiopia," Journal of Microbiology, Immunology and Infection, vol. 47, no. 4, pp. 333-338, 2014.

[19] S. Godreuil, F. Renaud, M. Choisy et al., "Highly structured genetic diversity of the Mycobacterium tuberculosis population in Djibouti," Clinical Microbiology and Infection, vol. 16, no. 7, pp. 1023-1026, 2010.

[20] J. Bruchfeld, G. Aderaye, I. B. Palme et al., "Molecular epidemiology and drug resistance of Mycobacterium tuberculosis isolates from ethiopian pulmonary tuberculosis patients with and without human immunodeficiency virus infection," Journal of Clinical Microbiology, vol. 40, no. 5, pp. 1636-1643, 2002.

[21] B. Diriba, T. Berkessa, G. Mamo, Y. Tedla, and G. Ameni, "Spoligotyping of multidrug-resistant Mycobacterium tuberculosis isolates in Ethiopia," International Journal of Tuberculosis and Lung Disease, vol. 17, no. 2, pp. 246-250, 2013.

[22] S. Verver, R. M. Warren, Z. Munch et al., "Transmission of tuberculosis in a high incidence urban community in South Africa," International Journal of Epidemiology, vol. 33, no. 2, pp. 351-357, 2004.

[23] J. R. Glynn, A. C. Crampin, M. D. Yates et al., "The importance of recent infection with Mycobacterium tuberculosis in an area with high HIV prevalence: a long-term molecular epidemiological study in Northern Malawi," Journal of Infectious Diseases, vol. 192, no. 3, pp. 480-487, 2005.

[24] P. J. Easterbrook, A. Gibson, S. Murad et al., "High rates of clustering of strains causing tuberculosis in Harare, Zimbabwe: a molecular epidemiological study," Journal of Clinical Microbiology, vol. 42, no. 10, pp. 4536-4544, 2004.

[25] C. Demay, B. Liens, T. Burguière et al., "SITVITWEB-a publicly available international multimarker database for studying Mycobacterium tuberculosis genetic diversity and molecular epidemiology," Infection, Genetics and Evolution, vol. 12, no. 4, pp. 755-766, 2012.

[26] I. C. Shamputa, L. Rigouts, L. A. Eyongeta et al., "Genotypic and phenotypic heterogeneity among Mycobacterium tuberculosis isolates from pulmonary tuberculosis patients," Journal of Clinical Microbiology, vol. 42, no. 12, pp. 5528-5536, 2004.

[27] O. Lahlou, J. Millet, I. Chaoui et al., “The genotypic population structure of Mycobacterium tuberculosis complex from Moroccan patients reveals a predominance of Euro-American lineages," PLoS ONE, vol. 7, no. 10, Article ID e47113, 2012.

[28] S. Gagneux, "Host-pathogen coevolution in human tuberculosis," Philosophical Transactions of the Royal Society B: Biological Sciences, vol. 367, no. 1590, pp. 850-859, 2012. 
[29] M. Coscolla and S. Gagneux, "Consequences of genomic diversity in Mycobacterium tuberculosis," Seminars in Immunology, vol. 26, no. 6, pp. 431-444, 2014.

[30] F. Cruciani, P. Santolamazza, P. Shen et al., "A back migration from Asia to sub-Saharan Africa is supported by highresolution analysis of human Y-chromosome haplotypes," American Journal of Human Genetics, vol. 70, no. 5, pp. 11971214, 2002. 

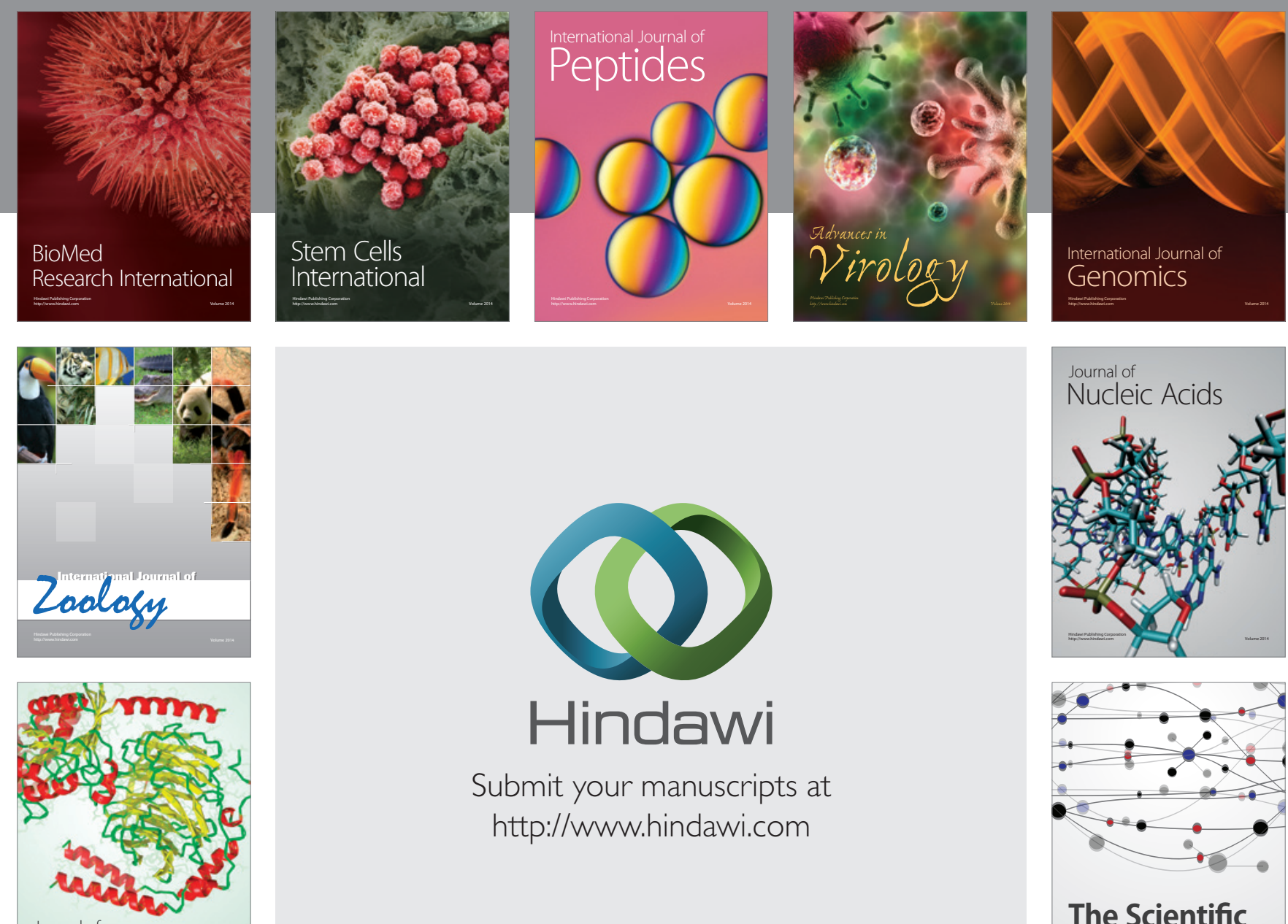

Submit your manuscripts at

http://www.hindawi.com

Journal of
Signal Transduction
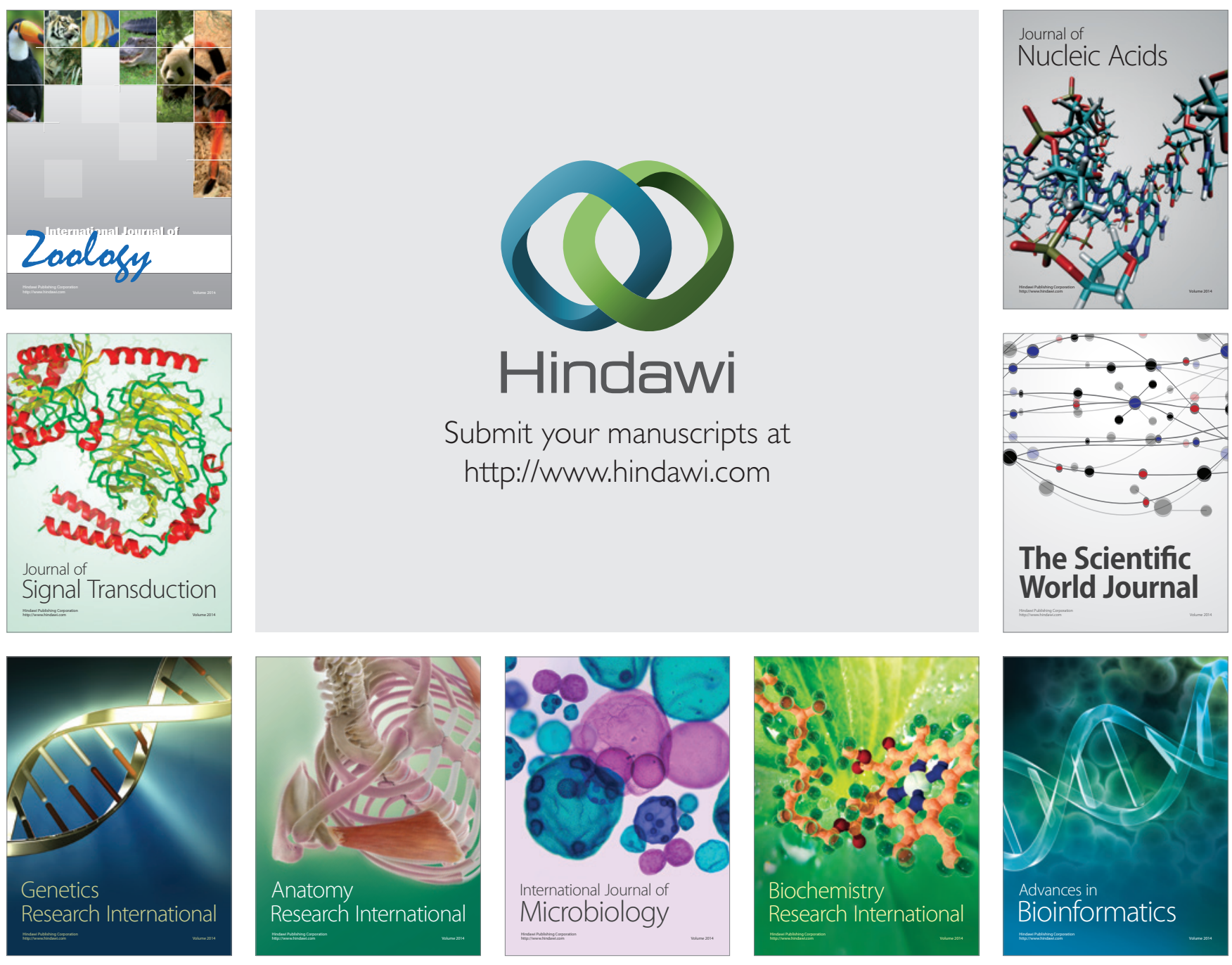

The Scientific World Journal
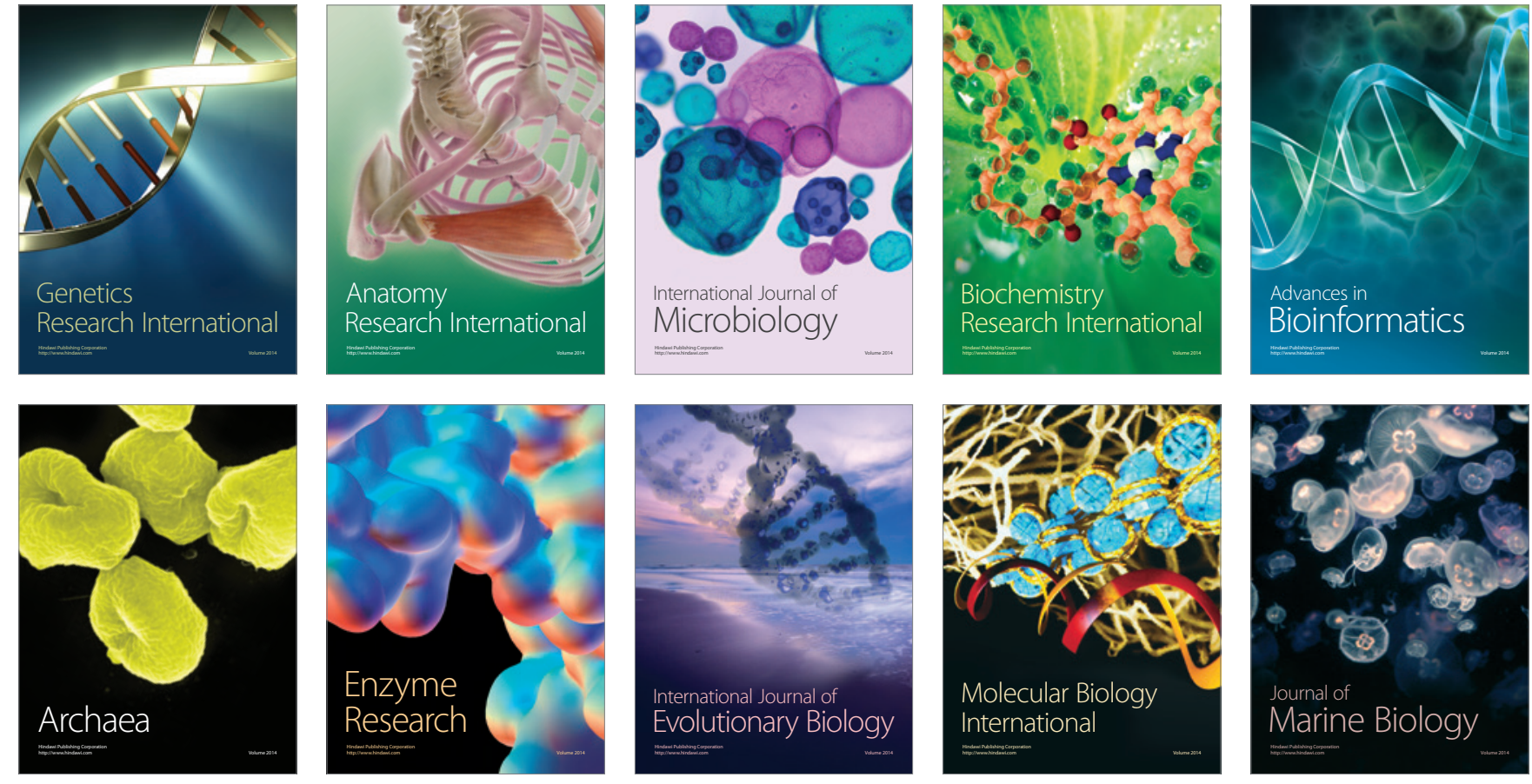